\title{
Los estilos de liderazgo de la Policía Local de la Comunidad Valenciana: Una aproxima- ción desde la Teoría del Liderazgo Transformacional
}

\author{
Octavio Álvarez*, Marisol Lila y Isabel Castillo
}

Universitat de València

\begin{abstract}
Resumen: Tomando como base la Teoría de Liderazgo Transformacional (Bass, 1985), se estudian las conductas que componen los estilos de liderazgo y su relación con tres variables de resultado: la eficacia del líder, la satisfacción con el líder y el esfuerzo extra de los subordinados; así como el aumento de los efectos (augmentation effect) del liderazgo transaccional cuando además se utiliza el liderazgo transformacional.

La muestra es representativa del universo de policías locales de la Comunidad Valenciana (España) y está compuesta por 975 policías locales (828 hombres y 147 mujeres) pertenecientes a las plantillas de 42 localidades y que son dirigidos por 42 jefes.

Los resultados muestran jefes con un perfil de liderazgo claramente activo, que combina conductas de liderazgo transformacional y liderazgo transaccional activo. No se han encontrado diferencias por género del subordinado que informa. Se confirma el poder predictivo positivo de los estilos transformacional y transaccional, y negativo de las conductas pasivas sobre las variables de resultado. Asimismo, se confirma el aumento de los efectos que el liderazgo transaccional posee sobre las variables de resultado analizadas, cuando además se utiliza el liderazgo transformacional. Se discuten los resultados y sus implicaciones prácticas.

Palabras clave: policía; seguridad pública; liderazgo transformacional; MLQ; satisfacción; eficacia; esfuerzo extra.
\end{abstract}

\section{Introducción}

En las últimas décadas el cuerpo de la policía local en España se ha ido transformando sensiblemente de forma que, de ser una institución cuya misión principal era mantener el "orden público", ha pasado a ser un organismo responsable de preservar la "seguridad ciudadana" (Jar, 1999). Así, la policía local, ha pasado a tener competencias en materia de seguridad ciudadana, tráfico, policía administrativa o policía judicial, por citar algunas (LO 2/1986). Igualmente, la policía local también ha evolucionado en cuanto a su formación, ampliándose considerablemente, tanto en lo que se refiere al tiempo de aprendizaje invertido, como en cuanto a la diversidad de materias en las que se forma a agentes y mandos (Garrido, Stangeland y Redondo, 2001). A modo de ejemplo, todos los policías locales de la Comunidad Valenciana deben realizar los cursos de acceso o promoción en el Instituto Valenciano de Seguridad Pública y Emergencias (IVASPE), recibiendo formación especializada de capacitación profesional.

Por otro lado, se recomiendan modelos policiales más proactivos frente a los reactivos más tradicionales. Estos modelos proactivos, como son la policía de proximidad, comunitaria o de barrio, pretenden minimizar el innegable

Dirección para correspondencia [Correspondence address]: Octavio Álvarez. Facultad de Psicología. Departamento de Psicología Social. Avda. Blasco Ibañez, 21. 46010 Valencia (España).

E-mail: octavio.alvarez@uv.es
Title: Leadership styles of Local Police in the Valencian Community: An approach based on the Transformational Leadership Theory.

Abstract: On the basis of the Transformational Leadership Theory (Bass, 1985), the patterns of behaviour found in leadership styles are examined, and also their relation to three outcome variables: leader efficacy, satisfaction with the leader and extra effort by subordinates. The study also deals with the augmentation effect in transactional leadership when combined with transformational leadership.

The sample is representative of the universe of local policemen in the Valencian Community (Spain) and it consists of 975 local policemen (828 men and 147 women) from 42 local municipalities and directed by 42 police chiefs.

Results show police chiefs with active leadership profile that combines transformational leadership behaviors and active transactional leadership. No differences were found by gender of subordinate informants. Both the positive predictive performance of the transformational and transactional styles and the negative predictive performance of passive behaviours were confirmed on the outcome variables. The results also confirm the increase of the transactional leadership effects on the outcome variables analysed when combined with transformational leadership. Practical implications are also discussed.

Key words: police; law enforcement; transformational leadership; MLQ; satisfaction; efficacy; extra effort.

componente reactivo de las intervenciones en la policía, posibilitando que el policía pueda actuar en la prevención como un profesional de la seguridad con conocimiento profundo de cada barrio y su cultura (Garrido et al., 2001; Torrente, 1999). De hecho, algunos autores han apuntado que "la policía comunitaria ha reinventado la Policía en cierto sentido" (Torrente, 1999, p. 90). Tanto los nuevos modelos policiales como la mayor formación de los subordinados demandan nuevas formas de dirección y liderazgo en la organización policial (e. g., Álvarez, 2005; Álvarez, Tomás, Falcó, Estevan, Molina-García, 2010b; Bynum, 2007, 2008; Downton, 1973; Morreale, 2003).

Una teoría de liderazgo que estudia cómo los estilos de liderazgo ayudan a transformar las organizaciones y los miembros que las componen, es la Teoría de Liderazgo Transformacional (Bass, 1985). Bass (1985) distingue entre tres estilos de liderazgo: transformacional, transaccional y laissez-faire o no liderazgo. El liderazgo transaccional es el estilo de liderazgo tradicional, es decir, el líder se relaciona con sus colaboradores en base a transacciones mediante las cuales éste ofrece incentivos para influir en el esfuerzo, así como una clarificación del trabajo necesario para obtener recompensas. Este estilo tradicional es necesario para un claro establecimiento de objetivos y la corrección de errores o desviaciones respecto de los objetivos establecidos. El estilo transformacional aumenta la influencia que el líder consigue con el estilo transaccional; los líderes transforman a sus seguidores haciéndoles más conscientes de la importancia y valor de los resultados de la tarea, activando sus necesidades de 
orden superior, e inculcándoles el interés personal trascendente por el bien del equipo. Como resultado de esta influencia, los seguidores sienten confianza y respeto hacia el líder, y están motivados a hacer más de lo que en un principio esperaban hacer. Por último, el estilo denominado laissezfaire o no liderazgo consiste en la ausencia de conducta de liderazgo donde el líder "elude la responsabilidad, retrasa decisiones, no proporciona feedback y no se interesa por satisfacer las necesidades de sus seguidores" (Northouse, 2001, p. 141). Tanto el estilo transformacional como el transaccio- nal están compuestos por conductas mostradas por el líder (Bass, 1985). Así, el estilo transformacional se compone de las conductas Influencia Idealizada que a su vez distingue la atribuida (atribución) y la observada (conducta), Motivación Inspiracional, Estimulación Intelectual y Consideración Individualizada y el estilo transaccional se compone de las conductas de Recompensa Contingente y Dirección por Excepción (activa y pasiva) (véase Tabla 1). (Para una revisión teórica reciente sobre liderazgo transformacional, véase Molero, 2011).

Tabla 1. Conductas que componen los estilos de liderazgo transformacional y transaccional.

\section{Conductas \\ Liderazgo Transformacional}

Influencia Idealizada

Motivación Inspiracional

Estimulación Intelectual

Consideración Individualizada

Liderazgo Transaccional

Recompensa Contingente

Dirección por Excepción

Descripción

Los jefes se convierten en modelos de sus subordinados. Son admirados, respetados y fidedignos. Se les atribuye por parte de los subordinados capacidades extraordinarias, persistencia y determinación. Se entiende que sus actos están guiados por hacer las cosas correctas, demostrando altos niveles de conducta ética y moral.

Los jefes encuentran maneras de motivar, proporcionando significados y retos a sus subordinados en el día a día. Se potencia el espíritu de equipo, llevando a los subordinados a implicarse en visiones atractivas de estados futuros. Crean expectativas sobre objetivos que los subordinados desean alcanzar con una comunicación clara, así como demuestran compromiso con los fines y la visión compartida.

Los jefes estimulan a los subordinados a esforzarse en ser innovadores y creativos, redefiniendo problemas y afrontando viejas situaciones de nuevas maneras. No hay críticas públicas de los errores individuales. Las nuevas ideas no son criticadas o eliminadas por diferir de las del jefe, al contrario, son alentadas.

Los jefes, en un clima de apoyo, prestan atención especial a cada necesidad individual de sus subordinados en cuanto al logro y el crecimiento, actuando como guía o mentor. Se reconocen las diferencias individuales en términos de necesidades y deseos. Los líderes aceptan las diferencias individuales (p. ej., algunos subordinados reciben mayor apoyo, más autonomía, otros estándares más firmes, y otros más dirección a la tarea). Se fomenta la comunicación bidireccional. Prima la consideración de "persona".

El jefe recompensa en el acto o promete recompensas a aquellos que trabajan en la dirección de los objetivos que se quieren cumplir.

Consiste en acciones correctivas que realiza el jefe. Estas acciones pueden ser activas, controlando los errores y actuando cuando se detectan, o pasivas, dejando que el subordinado cometa el error en su totalidad y actuando a posteriori.

Nota. Adaptado de Álvarez, Castillo y Falcó (2010a)

Tal y como plantea Bass (1985) en su Teoría del Liderazgo Transformacional, aunque los estilos de liderazgo transformacional y transaccional son procesos distintos, no son mutuamente excluyentes (Bass, 1985, 1998; Bass y Riggio, 2006) pudiendo ser utilizados por el líder en diferentes momentos y situaciones. De hecho, Bass (1985) propuso un modelo de relación entre ambos estilos de liderazgo, conocido como el "aumento del efecto" (angmentation effect por su denominación en inglés), sugiriendo que el liderazgo transformacional aumenta el efecto del liderazgo transaccional en la predicción de la satisfacción y otros resultados de los colaboradores. Concretamente, en terminología metodológica, el liderazgo transformacional aumenta la varianza explicada en valores de desempeño por encima de lo explicado por el liderazgo transaccional (e.g., Seltzer y Bass, 1987; Waldman, Bass y Yammarino, 1990). Asimismo, por medio del modelo Full Range de liderazgo eficaz, Avolio y Bass (1991) ponen en relación la eficacia del líder (de eficaz a ineficaz), la actividad de líder (de pasivo a activo) y la frecuencia con la que el líder exhibe cada estilo de liderazgo. Lo fundamental de este modelo es que todo líder muestra cada estilo en alguna medida
(Bass, 1998). Las relaciones entre estas variables dan como resultado dos perfiles: Por un lado encontramos al líder con un perfil óptimo que muestra, de menor a mayor frecuencia, conductas de laissez-faire, dirección por excepción pasiva, dirección por excepción activa, recompensa contingente, siendo las conductas de liderazgo transformacional las que exhibe con mayor frecuencia. Por otro lado encontramos al líder con un perfil sub-óptimo, el cual exhibiría la frecuencia de conductas inversa al anterior perfil, es decir, de menos a más frecuentemente, liderazgo transformacional, recompensa contingente, dirección por excepción activa, dirección por excepción pasiva y laissez-faire.

Son numerosos los estudios que emplean la Teoría del Liderazgo Transformacional para analizar el liderazgo en contextos tan diferentes como el industrial, militar y educativo (e.g., Bass, 1998; Castro Solano, 2006; Castro Solano y Benatuil, 2007; Wong, Bliese y McGurk, 2003); sanitario (e.g., Brown, 2003; Morales y Molero, 1995; Trofino, 2000), religioso (e.g., Brown, 2003; Druskat, 1994) o deportivo (Álvarez et al., 2010a), utilizándose en la gran mayoría de ellas el Cuestionario de Liderazgo Multifactorial (MLQ por 
sus siglas en inglés). En sus trabajos, autores como Densten (1999) utilizan como valores normativos los ofrecidos por Bass y Avolio (1990) procedentes de nueve estudios realizados en los contextos industrial, sanitario, educativo, militar, tanto del sector público como del privado. En general, en todos los contextos se han encontrando relaciones positivas entre el estilo de liderazgo transformacional y el compromiso y la satisfacción de los seguidores, la percepción de eficacia del líder y el esfuerzo extra según informan los subordinados (Bass y Riggio, 2006). Los líderes transformacionales se muestran más efectivos y satisfacen más como líderes que los transaccionales. Asimismo, el liderazgo transformacional aumenta el compromiso, la implicación, la lealtad y el desempeño de los subordinados, ayudando a manejar situaciones de estrés, mientras que el liderazgo transaccional induce estrés en mayor medida (Bass, 1999). No obstante, se han encontrado peculiaridades en algunos contextos específicos donde las conductas activas transaccionales de los líderes son percibidas como eficaces por los subordinados como, por ejemplo, en contextos militares con sistemas estables que cuentan con alta normativización (strong situations) (Antonakis, Avolio y Sivasubramaniam, 2003). En la misma línea, Castro Solano (2006) señala la importancia del contexto para la percepción de eficacia del líder, encontrando diferencias entre lo que informan muestras de civiles y de militares.

En cuanto al estilo de liderazgo y el género, las investigaciones realizadas al respecto informan de una heterogeneidad tanto de investigaciones como de resultados, difiriendo en función de las personas que realizan la evaluación (los propios líderes, los subordinados, o los compañeros), el tipo de organización, el instrumento de evaluación o el contexto cultural (véase Cuadrado, Molero y Navas, 2003). Así, la percepción del líder puede verse influida por los estereotipos de rol en función del género del subordinado que informa (Eagly, Makhijani y Klonsky, 1992). Por ejemplo, Cuadrado (2011) en su revisión sobre las diferencias por género en el liderazgo, señala que existen estudios que indican que los hombres tienen una mayor tendencia que las mujeres a evaluar como más eficaces a los líderes de su propio género ( $\mathrm{pa}-$ ra una revisión reciente sobre liderazgo y género véase Cuadrado, 2011).

La Teoría del Liderazgo Transformacional se ha puesto a prueba en el contexto policial (e.g., Álvarez, 2005; Álvarez et al., 2010b; Densten 2005; Murphy y Drodge, 2004). En su totalidad, los trabajos revisados apoyan el estilo de liderazgo transformacional como más eficaz en el contexto policial en la línea del modelo Full Range (Avolio y Bass, 1991). Por lo general, los líderes transformacionales tienen subordinados más satisfechos con el estilo del líder, lo perciben como un líder más eficaz y están dispuestos a ejercer un esfuerzo extra en su desempeño (e. g., Álvarez, 2005; Álvarez et al., 2010b; Morreal, 2003). En la misma línea, diversos estudios han mostrado relaciones positivas del liderazgo transformacional con otras variables como, por ejemplo, la motivación (Adebayo, 2005), los rasgos de personalidad (Rabstejnek,
2002), las actitudes en el trabajo (Dobby, Anscombe y Tuffin, 2004; Drodge y Murphy, 2002a, 2002b; Murphy y Drodge, 2004), la reducción del crimen, la percepción de seguridad por parte del ciudadano, la atención y las relaciones con el ciudadano (Vinzant y Crothers, 1994).

En suma, el liderazgo transformacional es el estilo de liderazgo que demuestra generar mayores niveles de satisfacción y esfuerzo extra del subordinado, y percepción de mayor eficacia del líder (Álvarez, 2005; Álvarez et al., 2010b; Avolio y Yammarino, 2002; Bass, 1998; Densten, 1999; Gasper, 1992; Lowe, Kroeck y Sivasubramaniam, 1996; Patterson, Fuller, Kester y Stringer, 1995). Así mismo, este liderazgo transformacional aumenta los efectos positivos del liderazgo transaccional sobre la satisfacción y el desempeño (Bass, 1985).

Por otra parte, autores como Bass y Riggio (2006) señalan que se necesita entender mejor si el liderazgo transformacional se ejerce en distintas culturas y diferentes grupos, y si bien existe literatura sobre fuerzas de seguridad en otros países, la literatura científica adolece de estudios de liderazgo en el contexto policial español (Álvarez, 2005).

Así pues, el objetivo del presente trabajo es múltiple. En primer lugar se estudian los estilos de liderazgo de los jefes de los policías locales de la Comunidad Valenciana (España), valorando si existen diferencias en la percepción del estilo de liderazgo de los jefes por parte de los subordinados en función del género del subordinado que informa. En segundo lugar, se realizará una comparación entre las medias obtenidas en el estudio y las medias que ofrecen Bass y Avolio (1990) como normativas. En tercer lugar, se analizará en qué medida estos estilos de liderazgo fomentan conductas de esfuerzo extra en los subordinados (relacionado con la motivación), satisfacción con la forma de liderar del jefe, haciendo que estos jefes sean percibidos como más o menos eficaces. Por último, se pondrá a prueba el aumento del efecto, es decir, si los efectos del estilo de liderazgo transaccional sobre las variables de resultado (esfuerzo extra, satisfacción y eficacia) se ven aumentados por el estilo de liderazgo transformacional.

\section{Método}

\section{Participantes y Procedimiento}

El estudio se llevó a cabo entre los meses de mayo y noviembre de 2007. Se elaboró un censo del universo de policías locales en la Comunidad Valenciana. Ya que el interés se centra en el liderazgo ejercido por policías, en este universo no se incluyeron las 69 localidades donde la jefatura era ejercida por un no policía (jefatura ejercida por el alcalde o por el concejal de seguridad). Asimismo y para mantener la confidencialidad de las localidades participantes en el estudio, se excluyeron las cinco localidades con jefatura ejercida por una mujer. Se realizó un muestreo estratificado aleatorio por conglomerados, por rango del jefe de la Policía local (o jefe del retén, en el caso de localidades con más de un retén) y 
género del sujeto que contesta, garantizando la representatividad de ambos estratos. La muestra por tanto ha sido estratificada por el rango del jefe, teniendo el 5.2\% como jefe un agente, el $17.9 \%$ un oficial, el $19.1 \%$ un inspector, el $19 \%$ un intendente, el $14.8 \%$ un intendente principal y el $24 \%$ tiene como jefe un intendente general.

La muestra final objeto de estudio está compuesta por 975 policías locales (828 hombres y 147 mujeres) que eran dirigidos por 42 jefes de policía. Esta muestra es representativa del universo de policías locales de la Comunidad Valenciana, pertenecientes a 42 localidades, con un error muestral de .03 .

Las edades de los participantes se distribuyen de la siguiente manera: el $8.3 \%$ tenía menos de 25 años, el 58.8\% tenía entre 25 y 35 años, el 19.4\% tenía entre 36 y 45 años, el $1.3 \%$ tenía más de 55 años, encontrando un $1.3 \%$ que no informó de su edad.

En las localidades se contactó telefónicamente con el jefe de la plantilla, explicándole el objeto de la investigación, quedando en ese momento para la visita y administración de cuestionarios. Todos los miembros de cada plantilla, a excepción del jefe, eran invitados a responder el cuestionario. La participación de todos los policías fue voluntaria y anónima.

\section{Instrumento}

Se administró la versión española (Molero, 1994; Molero, Recio y Cuadrado, 2010) del Cuestionario de Liderazgo Multifactorial (MLQ-5XC), Bass y Avolio, 1995) en la versión para subordinado. Este instrumento se compone de 45 ítems, 36 de los cuales recogen nueve conductas del líder con cuatro ítems cada una, la Influencia Idealizada (conducta), la Influencia Idealizada (atribución), la Motivación Inspiracional, la Estimulación Intelectual, la Consideración Individualizada, la Recompensa Contingente, la Dirección por Excepción Activa, la Dirección por Excepción Pasiva, y el Laissez-Faire; y los nueve ítems restantes recogen información sobre el Esfuerzo Extra del policía subordinado como resultado de las conductas del jefe (tres ítems), la Eficacia del jefe de policía como líder (cuatro ítems) y la Satisfacción con el jefe como líder (dos ítems). El cuestionario comienza con la frase: "Mi Jefe..." y las respuestas se recogen en una escala Likert con rango desde 0 (nunca) hasta 4 (casi siempre). Ejemplos de ítems serían: "Es efectivo a la hora de satisfacer mis necesidades en el trabajo", "Incrementa mi deseo de trabajar más", y "Es eficaz a la hora de cumplir las demandas de la organización".

\section{Resultados}

Se examinó la validez factorial del MLQ 5X (conductas de liderazgo y factores de resultado) a través del análisis factorial confirmatorio mediante el programa LISREL 8.54 (Jöreskog y Sörbom, 2003). Considerando la naturaleza ordinal de las variables latentes, se utilizó la matriz de correla- ciones policóricas y el método de mínimos cuadrados ponderados (Weighted Least Squares). Para medir la bondad de ajuste del modelo se utilizaron distintos índices (Hu y Bentler, 1999): $\chi^{2}$ dividido por los grados de libertad, el índice de ajuste no normativo (NNFI), el índice de ajuste comparativo (CFI), y la raíz del promedio del error de aproximación (RMSEA). Un cociente $\chi^{2} / \mathrm{gl}$ inferior a 5 representa un buen ajuste del modelo. Valores de CFI y NNFI por encima de .90 indican un ajuste aceptable. Para la RMSEA, se consideran aceptables valores entre .05 y .10 .

Para las conductas de liderazgo se hipotetizó la existencia de nueve variables latentes dependientes ${ }^{1}$, obtenidas en estudios anteriores (Bass y Avolio, 1990) correspondiente a las nueve conductas del líder (véase apartado instrumento) que subyacen a las 36 variables observables o ítems, y que dan cuenta de las covarianzas observadas entre ellas. Los resultados mostraron un adecuado ajuste de los datos: $\chi^{2} / \mathrm{gl}=4.06$ $\left[\chi^{2}(558)=2264.90, p=.00\right]$, CFI $=.98$, NNFI $=.98$, RMSEA $=.074(90 \%$ CI $=.071-.076)$, apoyando lo adecuado del modelo hipotetizado, así como saturaciones factoriales adecuadas (véase Tabla 2). La matriz phi mostró relaciones positivas entre las conductas que componen el liderazgo transformacional (rango entre .90 y .96), relación positiva entre las conductas que componen el liderazgo transaccional ( $\mathrm{r}$ $=.87$ ), así como entre las que componen el laissez faire $(\mathrm{r}=$ .98). Las relaciones fueron positivas entre las conductas del liderazgo transformacional y las del transaccional (rango entre .84 y .98) y negativas entre las conductas del liderazgo transformacional y las de laissez faire (rango entre -.72 y .82), así como entre las conductas del liderazgo transaccional y las de laissez faire (rango entre $-.71 \mathrm{y}-.78$ ).

Para los factores de resultado se hipotetizó la existencia de tres variables latentes dependientes: Esfuerzo Extra, Eficacia y Satisfacción que subyacen a las nueve variables observables, y que dan cuenta de las covarianzas observadas entre ellas. Los resultados mostraron un adecuado ajuste de los datos: $\chi^{2} / \mathrm{gl}=3.46\left[\chi^{2}(24)=83.13, p=.00\right]$, CFI $=.99$, $\mathrm{NNFI}=.99$, RMSEA $=.038(90 \% \mathrm{CI}=.030-.043)$, ароyando lo adecuado del modelo hipotetizado, así como saturaciones factoriales adecuadas (véase Tabla 3).

\footnotetext{
${ }^{1}$ Molero y colaboradores (2010) en la comprobación del ajuste de la estructura factorial y de otros posibles modelos alternativos utilizando una versión española del MLQ, encontraron que el modelo que presentaba el mejor ajuste era el formado por cuatro factores: liderazgo transformacional, liderazgo facilitador del desarrollo/transaccional, liderazgo correctivo y liderazgo pasivo/evitador. Como sugerencia de uno de los revisores, se ha puesto a prueba dicho modelo de cuatro factores presentando un aceptable ajuste a los datos: $\chi 2(588)=3918.39, \mathrm{p}=.00, \mathrm{CFI}=.98, \mathrm{NNFI}=.98, \mathrm{RMSEA}=.082$ $(90 \%$ CI $=.080-.085$, ECVI $=4.76$. Se utilizó el ECVI (Expected Cross Validation Index), para comparar los dos modelos alternativos. A medida que ECVI es más pequeño, o no varía entre los distintos modelos examinados, se entiende que el modelo se mantiene estable en la población. Comparando el ECVI del modelo de cuatro factores (4.76) con el ECVI de nueve factores (3.84), el segundo ajusta mejor a los datos. Además de por este motivo metodológico, hemos optado por mantener la estructura de nueve factores ya que fue la postulada teóricamente por los autores del MLQ, Bass y Avolio (1990).
} 
Tabla 2. Saturaciones factoriales estandarizadas del modelo de 9 conductas de liderazgo del MLQ.

\begin{tabular}{|c|c|c|c|c|c|c|}
\hline & & & licías I & & Norma & \\
\hline & Saturaciones factoriales & $\bar{M}$ & D.T. & $\alpha$ & MLQ Media & $t$ \\
\hline Influencia Idealizada - Conducta & & 2.23 & 1.00 & .81 & 2.64 & $11.06^{* *}$ \\
\hline MLQ6 & .53 & & & & & \\
\hline MLQ14 & .89 & & & & & \\
\hline MLQ23 & .77 & & & & & \\
\hline MLQ24 & .82 & & & & & \\
\hline Influencia Idealizada - Atribución & & 2.34 & 1.11 & .83 & 2.56 & $5.49 * *$ \\
\hline MLQ10 & .91 & & & & & \\
\hline MLQ18 & .63 & & & & & \\
\hline MLQ21 & .92 & & & & & \\
\hline MLQ25 & .68 & & & & & \\
\hline Motivación Inspiracional & & 2.46 & 1.14 & .90 & 2.64 & $4.37 * *$ \\
\hline MLQ9 & .80 & & & & & \\
\hline MLQ13 & .85 & & & & & \\
\hline MLQ26 & .91 & & & & & \\
\hline MLQ36 & .88 & & & & & \\
\hline Estimulación Intelectual & & 2.16 & 1.06 & .87 & 2.51 & $9.01 * *$ \\
\hline MLQ2 & .75 & & & & & \\
\hline MLQ8 & .77 & & & & & \\
\hline MLQ30 & .85 & & & & & \\
\hline MLQ32 & .87 & & & & & \\
\hline Consideración Individualizada & & 2.00 & 1.00 & .75 & 2.66 & $17.38^{* *}$ \\
\hline MLQ15 & .80 & & & & & \\
\hline MLQ19 & .51 & & & & & \\
\hline MLQ29 & .59 & & & & & \\
\hline MLQ31 & .91 & & & & & \\
\hline Recompensa Contingente & & 2.18 & 1.08 & .84 & 2.20 & 0.50 \\
\hline MLQ1 & .80 & & & & & \\
\hline MLQ11 & .62 & & & & & \\
\hline MLQ16 & .85 & & & & & \\
\hline MLQ35 & .85 & & & & & \\
\hline Dirección Excepción Activa & & 2.26 & 1.91 & .73 & 1.75 & $-15.14 * *$ \\
\hline MLQ4 & .72 & & & & & \\
\hline MLQ22 & .88 & & & & & \\
\hline MLQ24 & .47 & & & & & \\
\hline MLQ27 & .67 & & & & & \\
\hline Dirección Excepción Pasiva & & 1.52 & 1.00 & .70 & 1.11 & $-11.16^{* *}$ \\
\hline MLQ3 & .58 & & & & & \\
\hline MLQ12 & .78 & & & & & \\
\hline MLQ17 & .43 & & & & & \\
\hline MLQ20 & .92 & & & & & \\
\hline Laissez Faire & & 1.27 & 1.18 & .86 & 0.89 & $-9.24 * *$ \\
\hline MLQ5 & .83 & & & & & \\
\hline MLQ7 & .83 & & & & & \\
\hline MLQ28 & .87 & & & & & \\
\hline MLQ33 & .81 & & & & & \\
\hline
\end{tabular}

Nota. Todas las saturaciones son significativas, $t>1.96, p<.01$.

En las Tablas 2 y 3 se muestran las medias, desviaciones típicas y los coeficientes alfa de cada una de las conductas de liderazgo así como de los factores de resultado. Las conductas de liderazgo que en mayor medida se perciben son, por este orden, la Motivación Inspiracional y la Influencia Idealizada (atribución), correspondientes al estilo de liderazgo transformacional. Las conductas Dirección por Excepción Pasiva y Laissez-Faire son las que han obtenido la puntuación más baja, siendo por tanto, las que en menor medida se perciben. Asimismo los resultados muestran que la Eficacia percibida del líder es el factor de resultado que en mayor medida se percibe, seguido de la Satisfacción con el líder y el Esfuerzo Extra del colaborador. Los coeficientes de fiabilidad de las variables del estudio fueron satisfactorios (entre .70 y .92).

La comparación ( $t$ de Student) entre las medias obtenidas en el presente estudio y las medias que ofrecen Bass y Avolio (1990) como normativas en el MLQ-5X para cada uno de los factores de conducta, indicaron que en todas las conductas existen diferencias significativas $(p<.01)$, a excepción de 
la conducta de Recompensa Contingente $(p>.05)$. Los policías locales de la Comunidad Valenciana perciben en mayor medida conductas de Dirección por Excepción (activa y pasiva) y conductas de Laissez-Faire, y en menor medida el resto de las conductas en comparación con la norma ofrecida por Bass y Avolio (1990). De igual forma ocurre con los factores de resultado; estos factores indicaron diferencias estadísticamente significativas $(p<.01)$, puntuando más bajo en todos los factores los policías locales del presente estudio en comparación con los sujetos del estudio de Bass y Avolio (1990) (véase Tablas 2 y 3).

Tabla 3. Saturaciones factoriales estandarizadas del modelo de 3 factores de resultado del MLQ.

\begin{tabular}{|c|c|c|c|c|c|c|}
\hline & \multirow{2}{*}{$\begin{array}{c}\text { Saturaciones } \\
\text { factoriales }\end{array}$} & \multicolumn{3}{|c|}{ Policías Locales } & \multirow{2}{*}{$\begin{array}{l}\text { Norma } \\
\text { MLQ } \\
\text { Media } \\
\end{array}$} & \multirow[b]{2}{*}{$t$} \\
\hline & & $\bar{M}$ & $D T$ & $\alpha$ & & \\
\hline \multicolumn{7}{|l|}{ Esfuerzo Extra } \\
\hline MLQ39 & .87 & 2.03 & 1.29 & .92 & 2.60 & $11.75^{* *}$ \\
\hline MLQ42 & .94 & & & & & \\
\hline MLQ44 & .95 & & & & & \\
\hline Eficacia & & 2.39 & 1.14 & .88 & 2.62 & $5.78^{* *}$ \\
\hline MLQ37 & .91 & & & & & \\
\hline MLQ40 & .91 & & & & & \\
\hline MLQ43 & .88 & & & & & \\
\hline MLQ45 & .66 & & & & & \\
\hline Satisfacción & & 2.21 & 1.32 & .87 & 2.57 & $7.09 * *$ \\
\hline MLQ38 & .89 & & & & & \\
\hline MLQ41 & .91 & & & & & \\
\hline
\end{tabular}

Se realizaron análisis diferenciales por género $(t$ de Student), indicando que los varones y las mujeres policías locales del presente estudio perciben por igual tanto las conductas de liderazgo como el esfuerzo extra del colaborador, la eficacia percibida del líder y la satisfacción con el líder (véase Tabla 4).

Tabla 4. Descriptivos y diferencias por género del subordinado en las variables del estudio.

\begin{tabular}{|c|c|c|c|c|c|}
\hline & \multicolumn{2}{|c|}{ Varones } & \multicolumn{2}{|c|}{ Mujeres } & \multirow[b]{2}{*}{$t$} \\
\hline & $\bar{M}$ & $D T$ & $M$ & $D T$ & \\
\hline \multicolumn{6}{|l|}{ Conductas de Liderazgo } \\
\hline Influencia Idealizada-conducta & 2.22 & 1.00 & 2.28 & 1.02 & -0.64 \\
\hline Influencia Idealizada- atribución & 2.34 & 1.10 & 2.38 & 1.20 & -0.49 \\
\hline Motivación Inspiracional & 2.44 & 1.12 & 2.54 & 1.23 & -0.90 \\
\hline Estimulación Intelectual & 2.16 & 1.04 & 2.18 & 1.14 & -0.24 \\
\hline Consideración Individualizada & 2.00 & 0.98 & 2.00 & 1.09 & -0.07 \\
\hline Recompensa Contingente & 2.18 & 1.07 & 2.21 & 1.13 & -0.33 \\
\hline Dirección por Excepción (activa) & 2.26 & 0.91 & 2.26 & 0.94 & 0.04 \\
\hline Dirección por Excepción (pasiva) & 1.52 & 1.00 & 1.47 & 0.99 & 0.62 \\
\hline Laissez-Faire & 1.28 & 1.17 & 1.20 & 1.21 & 0.75 \\
\hline \multicolumn{6}{|l|}{ Factores de Resultado } \\
\hline Esfuerzo Extra & 2.03 & 1.27 & 2.07 & 1.39 & -0.36 \\
\hline Eficacia & 2.38 & 1.14 & 2.45 & 1.13 & -0.70 \\
\hline Satisfacción & 2.20 & 1.32 & 2.25 & 1.35 & -0.42 \\
\hline
\end{tabular}

Con la finalidad de estudiar en qué medida los estilos de liderazgo fomentan conductas de esfuerzo extra en los subordinados, satisfacción con la forma de liderar del jefe y eficacia de la conducta de dicho jefe se realizaron diversos análisis de regresión. Para garantizar la validez de los resultados del análisis de regresión, es necesario que se cumplan una serie de condiciones o supuestos (Pérez, 2004). Estos supuestos hacen referencia a la relación entre la variable dependiente y las independientes (linealidad); la relación entre las variables independientes (no colinealidad); las características de distribución y de los errores, teniendo en cuenta los criterios de independencia (estadístico de Durbin-Watson); homoscedasticidad (gráficos de dispersión de pronósticos y residuos tipificados); y normalidad (histograma y gráfico de probabilidad normal). Estos criterios o supuestos, fueron puestos a prueba, encontrando que todos se cumplían a excepción del supuesto de colinealidad entre las variables independientes, donde se obtuvo un Índice de Condición mayor de 15 , lo que sugería un posible problema de colinealidad (este problema es señalado por Bass (1999), como característico de las conductas que componen el estilo de liderazgo transformacional). El incumplimiento de este supuesto nos llevó a solucionar la colinealidad transformando las variables (Pérez, 2004). Esta transformación se llevó a cabo agrupando, según nos indica la teoría, las conductas de liderazgo en factores de orden superior formando lo que se ha etiquetado como estilos de liderazgo Transformacional, Transaccional y Laissez-Faire ${ }^{3}$. Siguiendo las indicaciones de Avolio, Bass y Jung (1997), el estilo de liderazgo transformacional está compuesto por las conductas Influencia Idealizada (conducta), Influencia Idealizada (atribución), Motivación Inspiracional, Estimulación Intelectual y Consideración Individualizada; el estilo de liderazgo transaccional está compuesto por las conductas Recompensa Contingente y Dirección por Excepción Activa; y por último, el estilo de liderazgo Laissez-Faire está compuesto por las conductas Dirección por Excepción Pasiva y Laissez-Faire. Los resultados del análisis factorial confirmatorio mostraron un adecuado ajuste de los datos: $\chi^{2} / \mathrm{gl}=4.54\left[\chi^{2}(582)=2644.24, p=.00\right]$, CFI $=$ $.98, \mathrm{NNFI}=.98, \mathrm{RMSEA}=.078(90 \% \mathrm{CI}=.075-.080)$, apoyando lo adecuado del modelo hipotetizado, así como saturaciones factoriales adecuadas. Los coeficientes alfa de Cronbach fueron de .96 para el estilo Transformacional, .86 para el estilo Transaccional y .88 para el estilo Laissez-Faire.

\footnotetext{
${ }^{3}$ Siguiendo las indicaciones de un revisor, se comparó los índices de ajuste del modelo hipotetizando nueve factores de orden inferior y tres factores de orden superior con el modelo de cuatro factores propuesto por Molero y colegas (2010). Se utilizó el ECVI para comparar los dos modelos alternativos (véase nota a pie de página anterior). El ECVI del modelo de seis factores de orden inferior y tres factores de orden superior fue de 4.27, y el ECVI del modelo de cuatro factores fue de 4.76, por lo que el primero es el que ajusta mejor a los datos.
} 
Tabla 5. Correlaciones bivariadas entre los estilos de liderazgo y los factores de resultado

\begin{tabular}{lllllll}
\hline & 1 & 2 & 3 & 4 & 5 & 6 \\
\hline Estilos de Liderazgo & & & & & & \\
1. Liderazgo Transformacional & - & & & & & \\
2. Liderazgo Transaccional & .90 & - & & & & \\
3. Laissez Faire & -.66 & -.61 & - & & & \\
Factores de Resultado & & & & & & \\
4. Esfuerzo Extra & .89 & .83 & -.63 & - & & \\
5. Eficacia & .88 & .82 & -.67 & .87 & - & \\
6. Satisfacción & .89 & .82 & -.67 & .89 & .88 & - \\
\hline
\end{tabular}

Nota. Todas las correlaciones son significativas $p<.01$

En la Tabla 5 se muestran las relaciones entre los tres estilos de liderazgo y los tres factores de resultado. Los estilos transformacional y transaccional se relacionan de forma positiva y significativa con los tres factores de resultado, mientras que el estilo laissez faire se relaciona de forma negativa y estadísticamente significativa con dichos factores.

Se analizó el poder predictivo de los tres estilos de liderazgo sobre las variables criterio Esfuerzo Extra, Eficacia y Satisfacción, mediante análisis de regresión múltiple (véase Tabla 6). El liderazgo Transformacional y el liderazgo Transaccional predijeron en sentido positivo y el estilo LaissezFaire en sentido negativo el esfuerzo extra de los subordina$\operatorname{dos}(F(3,971)=1301.61, p<.001)$, la eficacia de sus jefes $(F(3,971)=1281.03, p<.001)$ y la satisfacción con la forma de liderar su jefe $(F(3,971)=1292.28, p<.001)$; explicando cada ecuación el $80 \%$ de la varianza de cada una de las variables criterio.

Tabla 6. Análisis de Regresión Múltiple para predecir las variables de resultado

\begin{tabular}{|c|c|c|c|c|c|}
\hline & $B$ & Error $B$ & $\beta$ & $t$ & $R^{2}$ \\
\hline Esfuerzo Extra & & & & & .801 \\
\hline Liderazgo Transformacional & .93 & .05 & .70 & $19.92 * * *$ & \\
\hline Liderazgo Transaccional & .22 & .05 & .16 & $4.79 * * *$ & \\
\hline Laissez Faire & -.10 & .02 & -.08 & $-4.08 * * *$ & \\
\hline Eficacia & & & & & .798 \\
\hline Liderazgo Transformacional & .78 & .04 & .66 & $18.81 * * *$ & \\
\hline Liderazgo Transaccional & .17 & .04 & .14 & $4.10^{* * *}$ & \\
\hline Laissez Faire & -.17 & .02 & -.15 & $-7.83^{* * *}$ & \\
\hline Satisfacción & & & & & .800 \\
\hline Liderazgo Transformacional & .97 & .05 & .71 & $20.26 * * *$ & \\
\hline Liderazgo Transaccional & .14 & .05 & .09 & $2.82^{* *}$ & \\
\hline Laissez Faire & -.18 & .03 & -.14 & $-7.30 * * *$ & \\
\hline
\end{tabular}

Por último, se realizaron una serie de análisis de regresión jerárquica para analizar la hipótesis del aumento del efecto (Bass, 1985) del liderazgo transformacional sobre los tres factores de resultado (el esfuerzo extra de los subordinados, la eficacia de sus jefes y la satisfacción con la forma de liderar su jefe). El liderazgo transaccional se introdujo en el primer paso y el liderazgo transformacional en el segundo paso, para analizar si con esta introducción se encontraban cambios significativos en la $\mathrm{R}^{2}$ del modelo. Este acercamiento jerárquico nos ofrece información de cómo los cambios en la variable criterio están unidos a cambios en el estilo de liderazgo transformacional cuando la puntuación del liderazgo transaccional está controlada. Esto es, los coeficientes del segundo paso pueden ser considerados como la predicción de la varianza residual en cada variable de interés, no explicada por las respectivas variables iniciales. Los resultados mostraron que el liderazgo transformacional explica un incremento de la varianza del esfuerzo extra de los subordinados $\left(\Delta R^{2}=.11, p<.01\right)$, la eficacia de los jefes $\left(\Delta R^{2}=.11, p\right.$ $<.01)$ y la satisfacción con la forma de liderar del jefe $\left(\Delta R^{2}\right.$ $=.12, p<.01)$ (véase Tabla 7).

Tabla 7. Análisis de Regresión Jerárquica para explorar los efectos del aumento del liderazgo transformacional

\begin{tabular}{llllll}
\hline Paso & Variable & $\mathrm{R}^{2}$ & $\Delta \mathrm{R}^{2}$ & $\Delta F$ & $F$ \\
\hline & Esfuerzo Extra & & & & \\
1 & Liderazgo Transaccional & .69 & .69 & & $2206.44^{* *}$ \\
2 & Liderazgo Transformacional & .80 & .11 & 496.48 & $1913.25^{* *}$ \\
\hline & Eficacia & & & & \\
1 & Liderazgo Transaccional & .68 & .68 & & $2065.16^{* *}$ \\
2 & Liderazgo Transformacional & .79 & .11 & 479.76 & $1780.55^{* *}$ \\
\hline & Satisfacción & & & & \\
1 & Liderazgo Transaccional & .67 & .67 & & $1980.62^{* *}$ \\
2 & Liderazgo Transformacional & .79 & .12 & 543.55 & $1814.29^{* *}$ \\
\hline$* * p=.01$ & & & &
\end{tabular}

\section{Discusión}

El objetivo del presente trabajo ha consistido en estudiar, en una muestra de 975 policías locales representativos del universo de policías locales de la Comunidad Valenciana (España), los estilos de liderazgo de los jefes de policía local, atendiendo a las posibles diferencias por género del/a subordinado/a que informa. Se ha realizado una comparación entre las medias obtenidas en el estudio y las medias que ofrecen Bass y Avolio (1990) como normativas por el Cuestionario de Liderazgo Multifactorial (MLQ-5X@), Bass y Avolio, 1995). Se ha analizado en qué medida estos estilos de liderazgo fomentan conductas de esfuerzo extra en los subordinados (relacionado con la motivación), satisfacción con la forma de liderar del jefe, haciendo que estos jefes sean percibidos como más o menos eficaces. Por último, se ha puesto a prueba la hipótesis del aumento del efecto (augmentation effect $)$.

Las conductas de liderazgo de los jefes de policía que en mayor medida se presentan son la Motivación Inspiracional seguida de la Influencia Idealizada (atribución), la Dirección por Excepción Activa, la Influencia Idealizada (conducta), Recompensa Contingente, Estimulación Intelectual, Consideración Individualizada, Dirección por Excepción Pasiva, siendo las conductas de no liderazgo o Laissez-Faire las que en menor medida se presentan. Así pues, los resultados obtenidos nos presentan jefes que ejercen conductas de liderazgo mayoritariamente activas y transformacionales, utilizando estilos pasivos en menor medida. Autores como Antonakis y colaboradores (2003) señalan en esta línea que en contextos de alto riesgo (e. g., militares, bomberos, policías), la Dirección por Excepción Activa y, en general, las conduc- 
tas de liderazgo activo son evaluadas positivamente por los subordinados. Como explica Densten (1999), la policía debe reaccionar a las situaciones que generan muchos de los servicios (gran parte de las tareas de la policía es posterior a la ocurrencia de un hecho), lo que demanda Dirección por Excepción Activa teniendo gran control sobre recompensas y castigos por la propia naturaleza de la organización, realizadas por medio de conductas de Recompensa Contingente. Estos jefes, utilizan en menor medida la Estimulación Intelectual y Consideración Individualizada puesto que en las organizaciones policiales se tiende a fomentar el sentimiento de pertenencia al grupo frente al individualismo (Torrente, 1997). Este fomento de lo grupal frente a lo individual queda patente en los reglamentos, a modo de ejemplo, por medio de la uniformidad obligatoria, el saludo marcial frente a superiores, así como por medio de prácticas habituales no escritas, como el tratamiento de usted entre jefes y subordinados. Una explicación plausible de estos resultados, donde la Estimulación Intelectual y la Consideración Individualizada son ejercidas en menor medida que la conducta de Recompensa Contingente, nos la proporciona Vandenberghe (1999), quien señala que en contextos de alto estrés (como es el caso que nos ocupa), la Estimulación Intelectual puede fomentar sobrecarga en los subordinados, mientras que la Consideración Individualizada puede ser tomada como favoritismo o inequidad del líder hacia algún subordinado. El resto de conductas (Dirección por Excepción Pasiva y LaissezFaire) son las menos utilizadas por los jefes de Policía Local de la Comunidad Valenciana. A la luz de los descriptivos, los jefes de policía local encajarían en lo que Bass y colegas (p.e., Bass, 1998; Bass y Riggio, 2006) denominaron Liderazgo Carismático-Inspiracional (una combinación de las conductas Influencia Idealizada -conducta y atribución- con la Motivación Inspiracional) que ayudarían a fomentar sentimientos de cohesión, de misión de equipo.

Se ha realizado una comparación entre las medias obtenidas en el presente estudio y la norma ofrecida por Bass y Avolio (1990). Los policías locales presentan valores menores en todas las conductas de liderazgo, excepto en Dirección por Excepción Activa, Dirección por Excepción Pasiva y en Laissez-Faire. Estos resultados van en la línea de los obtenidos en el trabajo de Densten (1999), en el que se encuentra que todas las conductas de liderazgo en policías presentan valores por debajo de los de la norma (Bass y Avolio, 1990) a excepción de la conducta de Dirección por Excepción, perteneciente al estilo de Liderazgo Transaccional y la conducta de Laissez-Faire. En consonancia con la explicación que proporciona Densten (1999), consideramos que la policía opera con mecanismos que mantienen el status quo de sus miembros (los rangos y su poder legítimo), corrigiendo las desviaciones que se vayan produciendo. Los líderes en la Policía hacen hincapié en el control pero intervienen, preferiblemente, cuando las cosas van mal. El mismo Bass (1999) señala mayores niveles de conducta transaccional en el contexto militar. De forma similar, Antonakis y colaboradores (2003) informan de conductas de liderazgo activo, en parti- cular la Dirección por Excepción Activa, como conductas percibidas positivamente por los subordinados en contextos con un alto grado de normativización de las conductas. De igual forma, la labor policial tiene un componente reactivo innegable, puesto que en muchas ocasiones actúan posteriormente a la demanda de servicio del ciudadano.

En lo referente a los factores de resultado (Esfuerzo Extra, Eficacia del líder y Satisfacción con el líder), los resultados obtenidos señalan la Eficacia percibida del líder como el valor más alto, seguido de la Satisfacción con el líder, siendo el último el factor el Esfuerzo Extra. Comparando estos resultados con los de la norma (Bass y Avolio, 1990), los policías perciben como menos eficaces a sus superiores, están menos satisfechos con el estilo de liderazgo de sus superiores inmediatos y realizan conductas de esfuerzo más allá de lo esperable en menor medida que los subordinados del estudio normativo de Bass y Avolio (1990). Puesto que se ha comprobado que la presente muestra de policías presenta medias más bajas en las conductas transformacionales y más altas en las conductas Dirección por Excepción Activa, Dirección por Excepción Pasiva y Laissez-Faire que en el estudio de Bass y Avolio (1990), es esperable que las puntuaciones en las variables de resultado sean más bajas, ya que la literatura se sugiere que el estilo de liderazgo transformacional aumenta las variables de resultado (Esfuerzo Extra, Eficacia del líder y Satisfacción) con respecto al liderazgo transaccional (Bass, Avolio y Goodheim, 1987; Gasper, 1992; Hater y Bass, 1988; Patterson et al., 1995; Seltzer y Bass, 1987).

En suma, se puede trazar el siguiente perfil de líder en la Policía Local: tiene habilidad para motivar y construir equipos de trabajo, a través del espíritu de equipo, lo que otros autores han descrito como propia de las organizaciones policiales, esto es la solidaridad grupal (Reiner, 1978) y el compañerismo (Torrente, 1997). Nuestro líder en la Policía Local, utiliza su poder referente y experto como sistema de liderazgo, generando respeto y algún tipo de admiración en sus subordinados (Influencia Idealizada - atribución); ejerce la supervisión activa (Dirección por Excepción Activa), corrigiendo las desviaciones de las expectativas, fallos y errores en las tareas de sus subordinados; utiliza el modelado como sistema de liderazgo generando respeto y algún tipo de admiración en sus subordinados (Influencia Idealizadaconducta); refuerza las conductas de sus subordinados de manera contingente (Refuerzo Contingente); y fomenta el intercambio de ideas (Estimulación Intelectual).

Siguiendo el Modelo Full Range de Liderazgo (Avolio y Bass, 1991), se puede calificar el perfil obtenido por los jefes de la Policía Local de la Comunidad Valenciana como cuasisubóptimo claramente volcado hacia las conductas activas y empleando en menor medida las conductas pasivas.

La percepción del subordinado respecto a las conductas del líder puede verse influida por los estereotipos de rol (Eagly et al., 1992), por lo que se han estudiado las diferencias por género del/a subordinado/a que informa. Los resultados muestran que no existen diferencias por género en las percepciones de las conductas de los líderes, lo que puede 
ser debido a dos razones, bien que realmente no intervenga significativamente el factor del género de la/el policía subordinado o pudiera ser por la diferencia de muestra de varones frente a mujeres. Esta diferencia refleja fielmente la realidad del Cuerpo de Policía Local, mayoritariamente compuesto por hombres, hecho más acusado conforme se sube en el rango a evaluar (Martín, 1994; McConell, 2004; Silvestri 2003; Torrente, 1997).

Los resultados confirman las relaciones entre las conductas de liderazgo y las variables de resultado indicando que tanto los estilos de liderazgo transformacional como transaccional predicen de forma positiva y estadísticamente significativa el esfuerzo extra de los subordinados, la eficacia percibida del líder y la satisfacción con el líder. Por el contrario, el estilo de liderazgo Laissez-Faire predice de forma negativa y estadísticamente significativa dichas variables de resultado. Estos resultados van en la línea de lo postulado por Bass (1985) y confirmado en investigaciones revisadas posteriormente (Bass y Avolio, 1990; Bass et al., 1987; Densten, 1999; DeGroot, Kiker y Cross, 2000; Dumdum, Lowe y Avolio, 2002; Gasper, 1992; Hater y Bass, 1988; Lowe et al., 1996; Morreale, 2002, 2003; Panopoulos, 1998; Patterson et al., 1995; Seltzer y Bass, 1987).

Así mismo, se ha puesto a prueba la propuesta de Bass (1985) en la que sugiere que el liderazgo transformacional no es sustitutivo del liderazgo transaccional. Muy al contrario, el liderazgo transformacional aumenta los efectos del liderazgo transaccional activo sobre las variables de resultado. Los resultados de nuestro estudio van en la línea de trabajos anteriores (Howell y Avolio, 1993; Molero, Cuadrado, Navas y Morales, 2007; Seltzer y Bass, 1987; Waldman et al., 1990), obteniendo aumento en la varianza explicada de las tres variables de resultado cuando se añade el estilo de liderazgo transformacional al transaccional. En suma, los subordinados de nuestros jefes de la Policía Local, obtienen mayores niveles de satisfacción con el estilo de liderazgo del jefe, perciben como más eficaces a sus jefes y realizan conductas de esfuerzo extra con mayor probabilidad cuando sus jefes

\section{Referencias}

Adebayo, D. O. (2005). Perceived Workplace Fairness, Transformational Leadership and Motivation in the Nigeria Police: Implications for Change. International Journal of Police Science and Management, 7, 110-122.

Álvarez, O. (2005). Estilos de liderazgo: un estudio en una muestra de policías locales de la Comunidad Valenciana (trabajo de investigación sin publicar). Universitat de València.

Álvarez, O., Castillo, I. y Falcó, C. (2010a). Estilos de liderazgo en la Selección Española de taekwondo. Revista de Psicología del Deporte, 19, 219-230.

Álvarez, O., Tomás, I., Falcó, C., Estevan, I. y Molina-García, J. (2010b). Estilos de liderazgo: un estudio en una muestra de policías locales de la Comunidad Valenciana. VII Congreso Iberoamericano de Psicología. Actas del VII Congreso Iberoamericano de Psicología. CD-ROM. Oviedo, 20-24 Julio 2010.

Antonakis, J., Avolio, B. J. y Sivasubramaniam, N. (2003). Context and leadership: an examination of the nine-factor full-range leadership theory using the Multifactor Leadership Questionnaire. The Leadership Quarterly, 14, 261 295.

Avolio, B. J. (2005). Leadership Development in Balance: Made / born. Mahwah, New Jersey: Lawrence Erlbaum Associates, Inc., Publisehers.

Avolio, B. J. y Bass, B. M. (1991). The Full Range leadership development programs: basic and advanced manuals. Binghamton. NY: Bass, Avolio y Associates. adoptan estilos de liderazgo transformacionales además de transaccionales activos.

Para finalizar, quisiéramos indicar las implicaciones más relevantes que se desprenden del presente estudio. En primer lugar se comprueba que en el contexto de la policía en España, se cumplen también los supuestos de la teoría del Liderazgo Transformacional, dando así validez cross-cultural a dicha teoría. En segundo lugar, la evidencia empírica nos lleva a poder recomendar a los jefes, en el contexto policial, que si ejercen conductas de liderazgo transformacional, las consecuencias positivas que se derivan de un estilo de liderazgo transaccional activo se verán incrementadas en sus subordinados, invitando así a dirigir desde estilos de liderazgo más modernos (transformacionales) que respondan a nuevas realidades organizativas y sociales, con policías más formados y profesionalizados que mejorarán su percepción de eficacia, satisfacción y esfuerzo extra para con sus jefes.

Como limitación de este estudio quisiéramos señalar que no se han estudiado diferencias por género entre jefes de la Policía Local, debido a que en el momento de la investigación, las escasas localidades que contaban con Jefatura ostentada por una mujer hubieran impedido el anonimato de las plantillas y los jefes analizados.

Como futuras líneas de investigación, seguiremos a Avolio (2005) y Bass y Riggio (2006) quienes señalan la necesidad de más investigación sobre las relaciones lídersubordinado que arrojen luz sobre los procesos motivacionales implicados en el Liderazgo Transformacional. Asimismo apuntamos, en la línea de Densten (2003) y Avolio, Sosik, Jung y Berson (2003), la necesidad de acercamientos multinivel al estudio del liderazgo y que en este estudio en particular podría explicar la dispersión encontrada en las desviaciones típicas de las conductas de liderazgo. Por último, sería conveniente realizar estudios que contemplaran las diferencias-similitudes entre las autopercepciones de los jefes y de sus subordinados sobre los estilos y las conductas de liderazgo, así como el estudio de cómo puede influir la distancia organizacional entre el jefe evaluado y el subordinado que informa.

Avolio, B. J. y Yammarino, F. J. (2002). Transformational \& charismatic leadership: The road abead. Greenwich, CT: JAI

Avolio, B. J., Bass, B. M. y Jung, D. I. (1997). Replicated confirmatory factor analyses of the Multifactor Leadership Questionnaire. Binghamton, NY: Center for Leadership Studies, Binghamton University.

Avolio, B. J., Sosik, J. J., Jung, D. I. y Berson, Y. (2003). Leadership Models, Methods, and Applications. En W. Borman, D. R. Ilgen y R. K. Klimoski (Eds.), Handbook of Psychology: Volume 12 Industrial and Organizational Psychology (pp. 277-307). Hoboken, NJ: Wiley \& Sons

Bass, B. M. (1985). Leadership and performance beyond expectations. New York: Free Press.

Bass, B. M. (1998). Transformational leadership: industrial, military, and educational impact. Mahwah, NJ: Erlbaum.

Bass, B. M. (1999). Two Decades of Research and Development in Transformational Leadership. European Journal of Work and Organizational Psychology, 8, 9 -

Bass, B. M. y Avolio, B. J. (1990). Transformational Leadership Development: Manual for the Multifactor Leadership Questionnaire. Palo Alto, CA: Consulting Psychologists Press.

Bass, B. M. y Avolio, B. J. (1995). MLQ Multifactor Leadership Questionnaire: Sampler 
Set. Redwwod City. C. A.: Mind Garden.

Bass, B. M. y Riggio, R. E. (2006). Transformational Leadership. (2 $2^{\mathrm{a}}$ Ed.). Mahwah, NJ: Erlbaum.

Bass, B. M., Avolio, B. J. y Goodheim, L. (1987). Biography and the assessment of transformational leadership at the world class level. Journal of Management, 13,17-20.

Brown, M. G. (2003). Male leaders respond to the experience of changing leadership Expectations. (Doctoral Dissertation). Seattle University.

Bynum, R. (2007). Staff Education and Transformational Leadership in Criminal Justice. American Jails Magazine, 21, 28-30.

Bynum, R. (February 2008). Transformational Leadership and Staff Training in the Law Enforcement Profession. The Police Chief, 75, 72-87.

Castro Solano, A. (2006). Teorías implícitas del liderazgo, contexto y capacidad de conducción. Anales de Psicología, 22, 89-97.

Castro Solano, A. y Benatuil, D. (2007). Estilos de liderazgo, inteligencia y conocimiento tácito. Anales de Psicología, 23, 216-225.

Cuadrado, I. (2011). Liderazgo y discriminación femenina. En F. Molero y J.F. Morales (Coords), Liderazgo: becho y ficción (pp. 271-294). Madrid: Alianza Editorial.

Cuadrado, I., Molero, F. y Navas, M. (2003). El liderazgo de hombres y mujeres: diferencias en estilos de liderazgo, relaciones entre estilos y predoctores de variables de resultado organizacional. Acción Psicológica, 2, 2, 115-129.

DeGroot, T., Kiker, D. S. y Cross, T. C. (2000). A meta-analysis to review organizational outcomes related to charismatic leadership. Canadian Journal of $\mathrm{Ad}$ ministrative Sciences, 17, 356-371.

Densten, I. L. (1999). Senior Australian law enforcement leadership under examination. Policing: An International Journal of Police Strategies y Management, 22, 45-57.

Densten, I. L. (2003). Senior police leadership: does rank matter?. Policing: An International Journal of Police Strategies y Management, 26 (2), 400-418.

Densten, I. L. (2005). The Relationship Between Visioning Behaviours of Leaders an Follower Burnout. British Journal of Management, 16, 105-118.

Dobby, J., Anscombe, J y Tuffin, R. (2004). Police Leadership: Expectations \& Impact. Informe elaborado desde el Home Office Online Report 20/04. Recuperado el 2 de Julio de 2008 de http://www.homeoffice.gov.uk/rds/pubsstatistical.html

Downton, J. V. (1973). Rebel leadership style: Commitment and charisma in the revolutionary process. New York: The Free Press.

Drodge, E. N. y Murphy, S. A. (2002a). Police Leadership as a Transformational Social Process. International Journal of Police Science and Management, 3, 198-212.

Drodge, E. N. y Murphy, S. A. (2002b). Police Leadership Development: Building a Conceptual Framework. Gazette, 64, 35-39.

Druskat, D. U. (1994). Gender and leadership style: transformational and transactional leadership in the Roman Catholic Church. The Leadership Quarterly, 5 , 99-119.

Dumdum, U. R., Lowe, K. B. y Avolio, B. J. (2002). A meta-analysis of the transformational and transactional leadership correlates off effectiveness and satisfaction: an update and extension. En B. J. Avolio y F. J. Yammarino (Eds.), Transformational and charismatic leadership: the road abead (pp. 35-66). Amsterdam: JAI Press

Eagly, A.H., Makhijani, M.G. y Klonsky, B.G. (1992). Gender and the evaluation of leaders: A meta-analysis. Psychological Bulletin, 111, 3-22.

Garrido, V., Stangeland, P. y Redondo, S. (2001). Principios de criminologia. Valencia: Tirant lo blanch.

Gasper, S. (1992). Transformational leadershit: An integrative review of the literature. Doctoral Dissertation. Kalamazoo, MI: Western Michigan University.

Hater, J. J. y Bass, B. M. (1988). Superiors evaluations and subordinates' perceptions of transformational and transactional leadership. Journal of Applied Psychology, 73, 695-702

Howell, J. P. y Avolio, B. J. (1993). Transformational leadership, transactional leadership, locus of control, and support for innovation. Journal of Applied Psychology, 78, 891-902.

Hu, L. y Bentler, P. M. (1999). Cutoff criteria for fit indexes in covariance structure analysis. Conventional criteria versus new alternatives. Structural Equation Modeling, 6, 1-55.

Jar, G. (1999). El papel de la policía en una sociedad democrática. Revista Española de Investigaciones Sociológicas (Reis), 85. 199-220.

Jöreskog, K. y Sörbom, D. (2003). LISREL 8.54. Structural equation modeling with the Simplis command language. Chicago: Scientific Software International.

Lowe, K. B., Kroeck, K. G y Sivasubramaniam, N. (1996). Effectiveness correlates of transformational and transactional leadership: A meta-analytic review of the MLQ literature. Leadership Quarterly, 7, 385-425.

Martín, M. (1994). Mujeres policías. Madrid: CIS- Siglo XXI.

MCConnell, A. (2004). Lessons on leadership and promotion from female police executives. Doctoral Dissertation. Royal Roads University.

Molero, F. (1994). Carisma y Lideraggo carismático: una aproximación empírica desde las perspectivas de Bass y Friedman. Tesis Doctoral no publicada. Madrid: UNED.

Molero, F. (2011). Liderazgo transformacional y carismático. En F. Molero y J.F. Morales (Coords), Liderazgo: hecho y ficción (pp. 117-144). Madrid: Alianza Editorial.

Molero, F. Cuadrado, I., Navas, M. y Morales, J. F. (2007). Relations and Effects of Transformational Leadership: A Comparative Analysis with Traditional Leadership Styles. The Spanish Journal of Psychology, 10, 358-368.

Molero, Recio y Cuadrado (2010). Liderazgo transformacional y liderazgo transaccional: un anñalisis de la estructura factorial del Multifactor Leadership Questionnaire (MLQ) en una muestra española. Psicothema, 22, 495-501.

Morales, J.F. y Molero, F. (1995). Leadership in two types of healthcare organization. En J.M. Peiró, F. Prieto, J.L. Meliá y O. Luque (Eds.), Work and organizational psychology: European contributions of the nineties (pp. 209-221). East Sussex: Erlbaum.

Morreale, S. A. (2002). Transformational Leadership in Law Enforcement. En M. H. Vickers (Ed.), Proceedings of the 2002 Annual International Conference. New York: NY: aepp.net.

Morreale, S. A. (2003). Analysis of perceives leader behaviors in law enforcement agencies. Doctoral Dissertation. UMI, ProQuest Information and Learning Company. Ann Harbor, MI, USA.

Murphy, S. A. y Drodge, E. N. (2004). Four I's of Police Leadership: A Case Study Heuristic. International Journal of Police Science and Management, 6, 1-15.

Northouse, P. G. (2001). Leadership: Theory and practice (2nd ed.). Thousand Oaks, CA: Sage Publications.

Panopoulos, F. (1998). The Role of Transformational Leadership in a Follower Focused Model of Police Recruit Occupational Adjustment. Doctoral Dissertation. University of Sydney.

Patterson, C. Fuller, J. B., Kester, K. y Stringer, D. Y. (1995). A meta-analytic examination of leadership style and selected compliance outcomes. Paper presented at the Society for Industrial and Organizational Psychology, Orlando, FL.

Pérez, C. (2004). Técnicas de análisis multivariante de datos. Madrid: Pearson Educación S.A.

Rabstejnek, C. V. (2002). Prediction of transformational-transactional leadership from NEO PI-R FIVE-Factor Model of personality at senior military college. Doctoral Dissertation. UMI, ProQuest Information and Learning Company. Ann Harbor, MI, USA.

Reiner, R. (1978). The Blue-Coated Worker. Cambridge University Press.

Seltzer, J. y Bass, B. M. (1987). Leadership is more than initiation and consideration. New York, NY: American Psychological Association.

Silvestri, M. (2003). Women in Charge: Policing, Gender and Leadership. Cullompton, Devon: Willan Publishing, 2003.

Torrente, D. (1997). La sociedad policial. Barcelona: CIS.

Torrente. D. (1999). Prevención del delito y futuro de la policía. Revista Española de Investigaciones Sociológicas, 85, 85-94.

Trofino, A. J. (2000). Transformational leadership: moving total quality management to world-class organizations. International Nursing Review, 47, 232-242.

Vandenberghe, C. (1999). Transactional and Transformational Leadership: Suggestions for Future Research. European Journal of Work and Organizational Psychology, 8, 26-32.

Vinzant, J. y Crothers, L. (1994). Street-level leadership: The role of patrol officers in community policing. Criminal Justice Review, 19, 189-211.

Waldman, D. A., Bass, B. M. y Yammarino, F. J. (1990). Adding to contingent reward behavior: The augmenting effect of charismatic leadership. Group and Organizations Studies, 15, 381-391.

Wong, L., Bliese, P. y McGurk, D. (2003). Military leadership: A context specific review. The Leadership Quarterly, 14, 657-692.

(Artículo recibido: 06-04-2011; aceptado: 10-10-2011) 